\title{
Time dependent synthesis of gold nanoparticles using chitosan as reducing agent: A spectroscopic approach
}

\author{
K. R. SREELAKSHMI*, C. O. MOHAN, S. REMYA, REHANA RAJ, RADHIKA RAJASREE*, \\ DEVIKA PILLAI*, K. ASHOK KUMAR AND C. N. RAVISHANKAR \\ ICAR-Central Institute of Fisheries Technology, Matsyapuri. P. O., Willigdon Island, Kochi, Kerala, India \\ *Kerala University of Fisheries and Ocean Studies, Panangad, Kochi-682 506, Kerala, India \\ e-mail: sreecift@gmail.com
}

\begin{abstract}
The present study attempted synthesis of gold nanoparticle using chitosan from shrimp waste and evaluated the kinetics of gold nanoparticle (AuNPs) formation. Two different forms of chitosan (high molecular weight and medium molecular weight forms) were used for the preparation of AuNPs. The formation of nanoparticles was studied using UV-VIS spectra obtained at different time intervals during synthesis. The typical UV-VIS spectrum of non-aggregated spherical AuNPs at $520 \mathrm{~nm}$ was obtained for both chitosan forms and the maximum absorbance was higher for the sample prepared with medium molecular weight chitosan. The results clearly indicated that the characteristic surface plasmonic resonance (SPR) peak became recognisable at $5 \mathrm{~min}$ of heating for the sample with high molecular weight chitosan and 3 min of heating for medium molecular weight chitosan. This indicated that, the lower the molecular weight, the faster the formation of nanoparticles. The wavelength $(\lambda)$ of absorption maxima was selected and normalised with respect to the absorption maxima at $15 \mathrm{~min}$. The sigmoidal shape of kinetic curve implied the autocatalytic character of AuNP formation. The data from the samples were fitted with two step Finke-Watzky kinetics model and the kinetics constants were derived. The rate constant of the nucleation reaction was found higher for the medium molecular weight chitosan.
\end{abstract}

Keywords: AuNPs, Chitosan, Gold nanoparticles, Molecular weight, Rate constant

\section{Introduction}

Gold nanoparticles (AuNPs) attract considerable interest in various fields of medicine, photonics, material science and chemistry due to their novel physical and chemical characteristics likeopticaland catalytic properties. Nanoparticles can be synthesised using top-down or bottom-up strategies. The size of large materials are broken down in top-down approach while in bottom-up approach, nanometer scale molecular structures are produced by assembling smaller particles like atoms or molecules (Mazzola, 2003). The second strategy is used commonly for the biological and chemical synthesis of nanoparticles. Metal nanoparticles are generally synthesised and stabilised with electrochemical techniques, chemical methods, mechanical methods, photochemical reactions as well as green chemistry methods. It is possible to develop nanoscale systems using AuNPs which interact with biological and chemical systems. The most efficient and simpler technique is chemical reduction and involves the reduction of $\mathrm{Au}(\mathrm{III})$ to gold with an oxidation state of zero but these chemicals are harmful to the environment limiting the applications.

Another significant approach in AuNPs synthesis includes biosynthesis and green technologies. Biosynthesis can be done using microbes like fungi, bacteria and marine algae and the process can be undertaken using intracellular or extracellular techniques. This technique is eco-friendly, inexpensive and time saving (Patil and Kim, 2018). Green technologies involve the use of natural extracts or polysaccharides for the reduction of gold precursor. The Van der Waals' interactions cause the nanoparticles to aggregate and reduce dispersion. Many polysaccharides like chitosan, alginate, gum arabic and starch can act as effective stabilising agents.

Chitin is the second most abundantly available biopolymer which is present in fungi, algae, shrimps and crabs and is a linear polymer of $\mathrm{N}$ acetyl-Dglucosamine. The shells of shrimps and crabs form one of the important sources for commercial extraction. The deacetylation of chitin yields chitosan, a high molecular weight linear polymer of amino-D-glucose. It is non-toxic and is used in food and agricultural applications. The biodegradability, biocompatibility and bioactivity of chitosan has led to various applications in biomedical and pharmaceutical industries (Osman and Arof, 2003). It is available with varying degrees of deacetylation and with different molecular weights as a result of the chemical depolymerisation which is very important in deciding its 
applications. Studies have indicated its efficacy as both reducing agent and stabilising agent for the formation of metal nanoparticles (Huang and Yang, 2004).

The future of nanotechnology lies in improved understanding of the kinetics and thermodynamics of nanoparticle synthesis, growth and aggregation (Dutta et al., 2016). The kinetics of nanoparticle formation depends on various factors like temperature of treatment, time and reagents used for synthesis. Although previous reports are available on the synthesis of AuNPs using chitosan (Huang and Yang, 2004; Mohan et al., 2019) there is no literature available on the kinetics of synthesis of gold nanoparticles as affected by different characteristics of chitosan. Hence the present work was undertaken to study the kinetics of AuNPs synthesis using chitosan having two different molecular weights.

\section{Materials and methods}

Two different forms of chitosan viz., Chitosan 1 (high molecular weight) and Chitosan 2 (medium molecular weight), extracted from shrimp processing waste were procured from ISF, Chitin and Marine products LLP at Kochi, Kerala, India. Hydrogen tetrachloroaurate (III) trihydrate and acetic acid were obtained from Merck Life Science Ltd., India. All solutions were prepared using milli $\mathrm{Q}$ water.

\section{Synthesis of gold nanoparticles}

Chitosan was dissolved in $1 \%$ acetic acid (w/v) at a concentration of $0.2 \%$ and heated at $90^{\circ} \mathrm{C}$ for $30 \mathrm{~min}$ with continuous stirring at $500 \mathrm{rpm}$. Gold precursor, auric chloride $(10 \mathrm{mM})$ was added to the chitosan solution and heated further for $15 \mathrm{~min}$. This was done separately with both forms of chitosan (Chitosan 1 and 2). During thermal treatment, the colour of the solution changed from light yellow to pink on synthesis of gold nanoparticles. With increase in time of treatment, the colour turned to ruby red.

\section{UV-VIS spectroscopy}

The UV-VIS spectra of the solutions were monitored using Jasco Dual Beam Spectrophotometer (Model V-570; Jasco International Pvt Ltd., Japan). The solutions were characterised for UV spectra at an interval of one minute. UV-VIS spectra of chitosan solutions were taken over the wavelength of 200-700 nm and $300-700 \mathrm{~nm}$ after addition of gold precursor (Wang et al., 2017). The solutions were used directly for spectroscopic analysis without any dilution. The data obtained was fitted using two step Finke-Watsky kinetics model to study the synthesis of nanoparticles and the kinetics constants were derived (Watsky and Finke, 1997).

\section{Transmission electron microscopy (TEM)}

The physical properties of AuNPs synthesised using the two different forms of chitosan were evaluated using transmission electron microscopic (TEM) images. TEM analysis was done in a transmission electron microscope (JEM 2100; Jeol, Japan) at $200 \mathrm{kV}$. The AuNP solutions prepared using chitosan of $0.2 \%$ concentration were dropped on a carbon-coated copper grid and allowed to dry at room temperature. The emission source was lanthanum hexaboride single crystal source and had a resolution of $0.23 \mathrm{~nm}$ (point) and $0.14 \mathrm{~nm}$ (lattice).

\section{Results and discussion}

The important chromophoric groups in chitosan are $\mathrm{N}$-acetyl glucosamine and glucosamine. The UV spectra of the chitosan used in the study (Fig. 1) showed bands with distinguished peak around $300 \mathrm{~nm}$. The intensity of the peak varied with the two forms of chitosan and the higher molecular weight chitosan had higher maximum absorbance. According to Tyagi et al. (1996), pure chitosan has two bands at 210 and $260 \mathrm{~nm}$. The red shift of the bands can be the resultant of interaction of acetic acid and chitosan as acetic acid was used to dissolve chitosan.

Pure chitosan solution dissolved in acetic acid was almost colourless and the addition of gold chloride under thermal treatment gave pink colour on formation of AuNPs. The intensity of colour increased with treatment time (Fig. 2). The protonation of amine groups of chitosan in acidic solution enables the dissolution of chitosan and the ionic repulsion between these amine groups gives an extended linear configuration to chitosan. Studies have indicated the presence of amino, ester and hydroxyl groups in chitosan making it a stable material for supporting nanoparticles preventing its aggregation (Rinaudo, 2006). According to Sun et al. (2017), three major reactions are involved in the formation of AuNPs in chitosan solution:

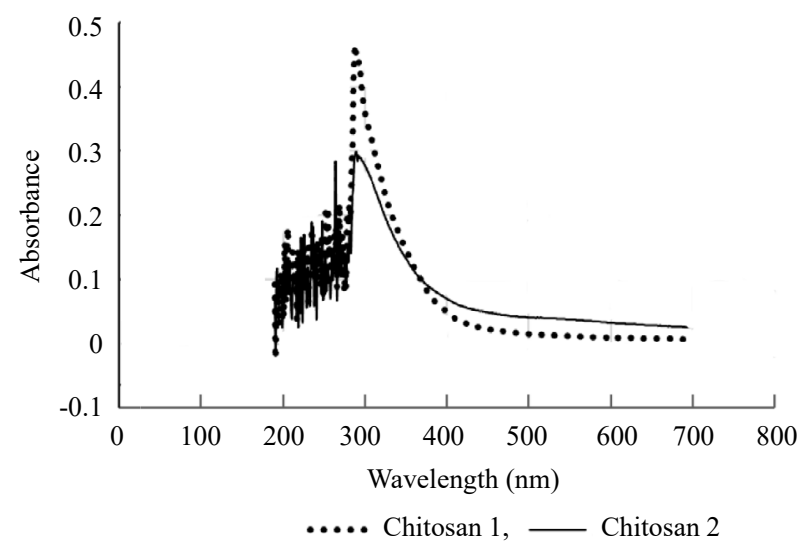

Fig. 1. UV-VIS spectra of chitosan 1 and chitosan 2 


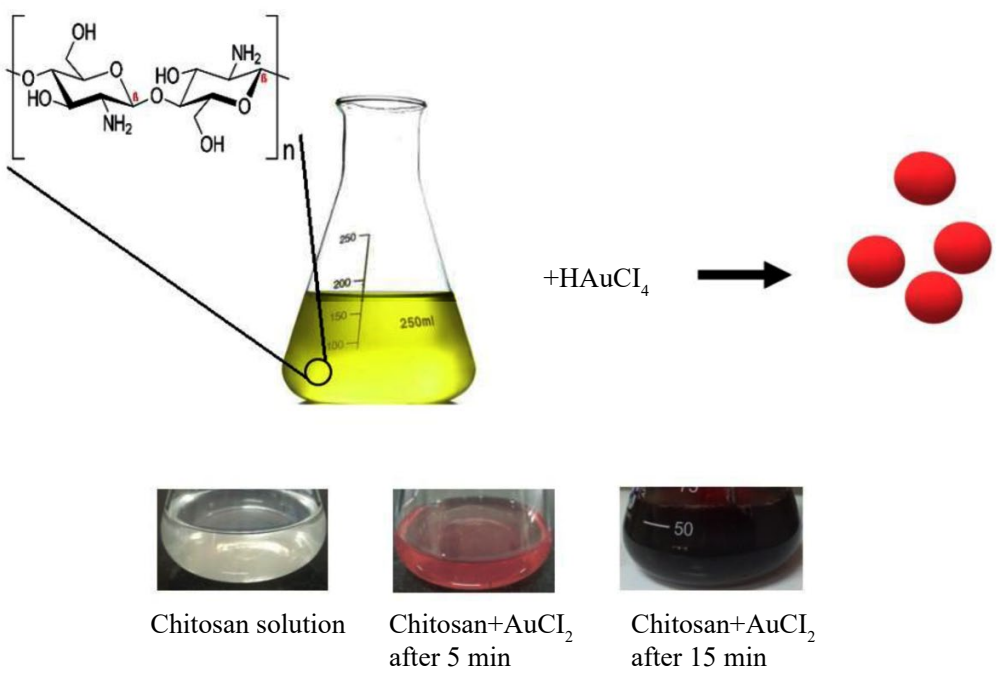

Fig. 2. Pictorial representation of synthesis of chitosan gold nanocomposites

(1) protonated $\mathrm{NH}_{2}$ groups in chitosan and $\mathrm{AuCl}_{4}$ ions undergo electrostatic interaction; (2) in chitosan, the $-\mathrm{CHO}$ groups at $\mathrm{C}_{1}$ position and the $-\mathrm{CH}_{2} \mathrm{OH}$ groups at $\mathrm{C}_{6}$ position undergo oxidation with simultaneous reduction of $\mathrm{Au}^{3+}$ ions and (3) the coulombic and covalent interactions cause the absorption of gold hydroxychlorides. Reports have suggested that heating also aids in the reduction of gold ions to nanoparticles (Akhtar et al., 2013). The major reduction reaction, represented by Lakshmi Narayanan and Shivakumar (2014) is as follows:

$$
\mathrm{AuCI}_{3}(\mathrm{aq})+3 \mathrm{e}^{-}+3 \mathrm{NH}^{+} \rightarrow \mathrm{Au}^{0} 3 \mathrm{NH}_{2}+3 \mathrm{HCI}
$$

The surface plasmonic resonance (SPR) peak of AuNPs is influenced by the morphology and physicochemical environment of AuNPs. The typical UV-VIS spectrum of non-aggregated spherical AuNPs is composed of a band of surface plasmonic resonance at $520 \mathrm{~nm}$ and a shorter wavelength absorption edge formed by the transitions of d-band electrons (Amendola and Meneghetti, 2009). The UV-VIS spectra of AuNPs were measured by taking samples from the reaction mixture during the course of synthesis (Fig. 3a and 4a). Both chitosan samples were able to reduce gold ions to nanoparticles which is evident from the SPR peak. The results clearly indicated that the characteristic SPR peak became recognisable after 5 min of heating for chitosan 1 and at $3 \mathrm{~min}$ of heating for chitosan 2 . This showed that the formation of nanoparticles was faster with medium molecular weight chitosan. The SPR peak became more pronounced in all the samples with increase in time of heating which indicated positive relationship between heating time and the number of nanoparticles formed. As the characteristics of the SPR peak is sensitive to the shape and size of
AuNPs, the increase in the absorption maximum with time corresponds to growth of nanoparticles (Mulvaney, 1996). The absorbance maxima was comparatively higher for the AuNPs synthesised using medium molecular weight chitosan. The higher availability of the reducing ions can cause increased formation of AuNPs (Anthonsen et al., 1994). No red or blue shift was observed in the absorbance maxima of the two samples, which indicated no difference in the chitosan units surrounding the AuNP surface.

From the UV-VIS absorption data, the wavelength $(\lambda)$ of absorption maxima was selected and the absorption maxima at the selected $\lambda$ as a function of time $t$ was normalised with respect to the absorption maxima at $\mathrm{t}=15$ min. The kinetic curve plotted with absorption maxima of the samples had a sigmoidal shape (Fig. $3 b$ and $4 b$.). The sigmoidal shape of kinetic curve implies the autocatalytic character of AuNP formation which can be explained by the Finke-Watzky model (Watzky and Finke, 1997). The data from all the samples were fitted with two step Finke-Watzky kinetics model and the kinetics constants were derived.

The Finke-Watzky model has two consecutive steps: First a homogeneous nucleation process resulting in the formation of gold nuclei which is a slow and continuous process with rate constant $k_{1}$.

$$
\mathrm{A} \rightarrow \mathrm{B}
$$

Second step is the autocatalytic growth of nanoparticles with rate constant $k_{2}$.

$$
\mathrm{A}+\mathrm{B} \rightarrow 2 \mathrm{~B}
$$

Both nucleation and growth of nanoparticles fitted to the model with an $\mathrm{R}^{2}$ value of 0.99 . The rate constant 


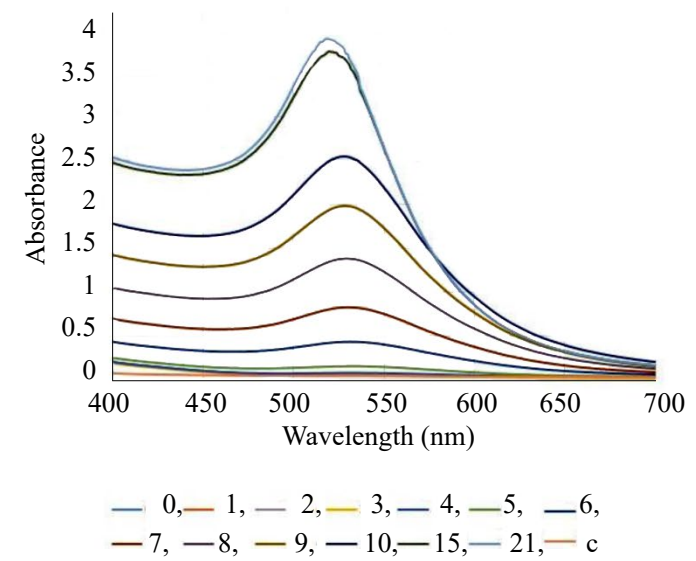

(a)

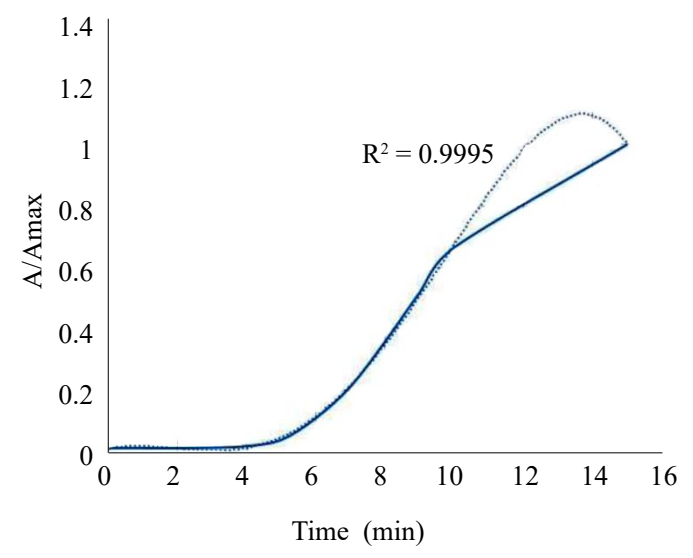

(b)

Fig. 3. (a) UV-VIS spectra of chitosan 1-AuNP nanocomposites measured during synthesis. (b) Normalised absorption maxima with dotted lines representing Finke-Watsky fit.

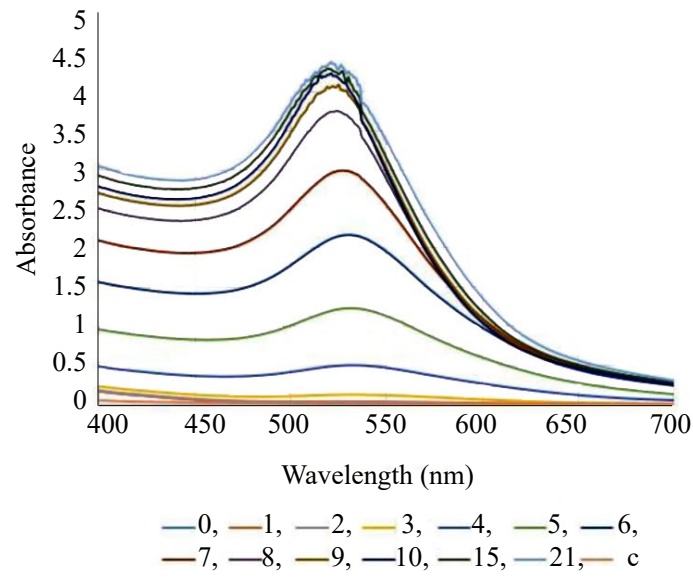

(a)

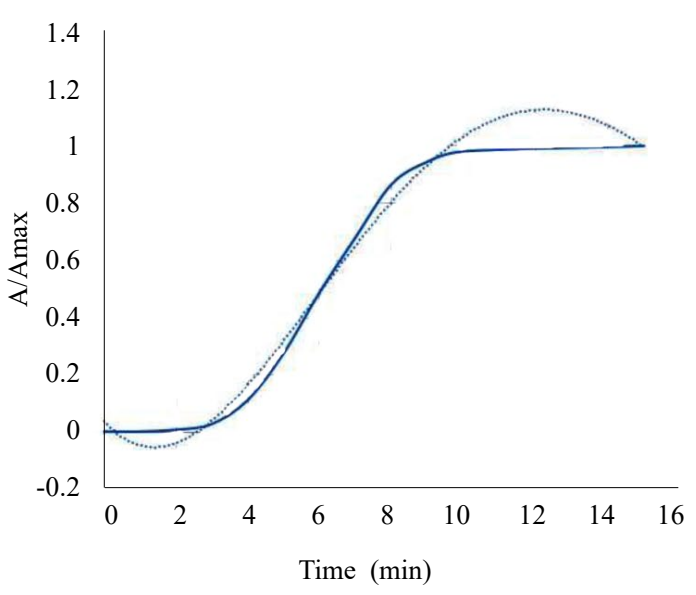

(b)

Fig. 4. (a) UVVIS spectra of chitosan 2-AuNP nanocomposites measured during synthesis. (b) Normalised absorption maxima with dotted lines representing Finke-Watsky fit

of the nucleation reaction was higher when medium molecular weight chitosan was used. With increase in molecular weight, the intermolecular hydrogen bonding also increases and this activates entangling. The reduction capacity of the groups in low molecular weight chitosan chain is higher because of the extended structure, making the nucleation process faster. The degradation of chitosan changes the conformation of chitosan and increases the glucosamine units, which in turn leads to increase in $\mathrm{CHO}$ groups, thereby enhancing the reducing ability for reducing gold ions (Sun et al., 2017). The rate constant of growth of nanoparticles was more than 18 times faster than the nucleation process in both the samples studied (Table 1). Simeonova et al. (2018) reported $k_{1}$ of $0.007 \mathrm{k}_{1} \mathrm{~s}^{-1}$ and $k_{2}$ of $0.39 \mathrm{k}_{2} \mathrm{M}^{-1} \mathrm{~s}^{-1}$ for nanoparticles synthesised using citric acid and chitosan. The classical Turkevich method studied using $2.5 \mathrm{mM}$ gold precursor had given $k_{1}$ of $0.0047 \mathrm{k}_{1} \mathrm{~s}^{-1}$ and $k_{1}$ of $0.36 \mathrm{k}_{2} \mathrm{M}^{-1} \mathrm{~s}^{-1}$ (Georgiev et al., 2013).

The electron microscopic observation of the two samples (Fig. 5) clearly indicated the formation of AuNPs. Higher molecular weight chitosan resulted in larger nanoparticles in comparison to the medium molecular weight chitosan. It was also evident that most of the nanoparticles formed pertained to a spherical shape but a few indicated anisotropic growth leading to the formation of nanoparticles of different shapes.

The present study aimed to utilise chitosan, a potent bioactive compound from shrimp waste for the synthesis 
Table 1. The rate constants of chitosan

\begin{tabular}{|c|c|c|c|c|c|c|c|}
\hline Chitosan & $\begin{array}{l}\text { Concentration of } \\
\text { chitosan }(\%)\end{array}$ & $\begin{array}{l}\text { Degree of deacetylation } \\
\text { (DDA) }\end{array}$ & Maximum absorbance & $k_{1} \mathrm{k}_{1} / \mathrm{s}$ & $k_{2} \mathrm{k}_{2} / \mathrm{Ms}$ & $k_{2} / k_{1}$ & $\mathrm{R}^{2}$ \\
\hline Chitosan 1 & 0.2 & 84.3 & 3.737 & $1.39 * 10^{-3}$ & 0.11 & 72.55 & 0.9995 \\
\hline Chitosan 2 & 0.2 & 84.3 & 4.299 & $8.30 * 10^{-3}$ & 0.15 & 18.59 & 0.9908 \\
\hline
\end{tabular}

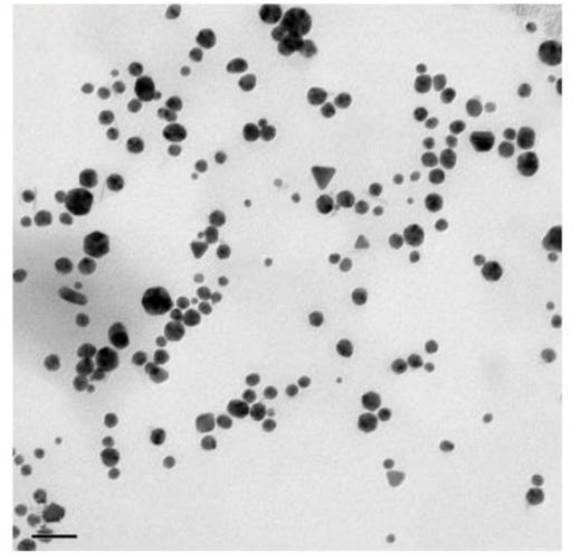

(a)

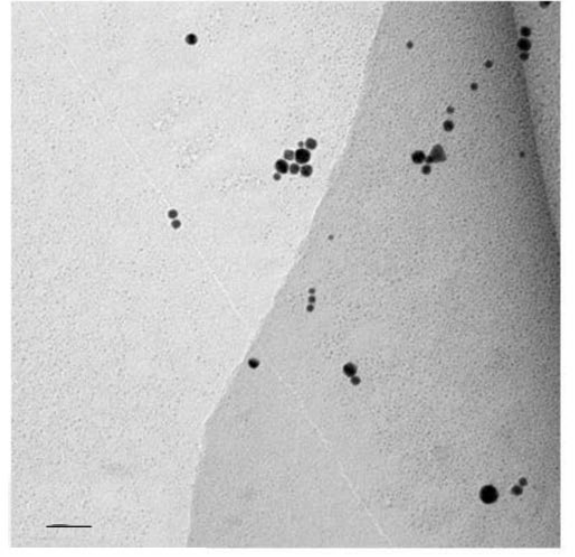

(b)

Fig. 5. Transmission electron microscopic images of AuNPs (a) Chitosan 1 and (b) Chitosan 2

and characterisation of AuNPs. In the study, the AuNPs were synthesised using chitosan of two different molecular weights. Spectroscopic data was collected throughout the synthesis period and the effect of chitosan on nanoparticle synthesis was studied using the Finke-Watzky model. The formation of AuNPs using both the forms of chitosan resulted in typical nucleation and growth phase with sigmoidal fit. The rate constants obtained were compared and was seen that the lower the molecular weight, the higher was the rate constant of nucleation process. The results of the present study can be used as baseline data in selecting suitable quality of chitosan for synthesis of gold nanoparticles as per the required applications.

\section{Acknowledgements}

Authors would like to thank the Director, ICARCIFT, Kochi for granting permission to conduct this study and publish this work. First author would like to thank the Dean, College of Fisheries, KUFOS, Panangad for granting Ph. D. admission.

\section{References}

Akhtar, M. S., Panwar, J. and Yun, Y. S. 2013. Biogenic synthesis of metallic nanoparticles by plant extracts. ACS Sustain. Chem. Eng., 1(6): 591-602. https://doi.org/10.1021/sc 300118u.

Amendola, V. and Meneghetti, M. 2009. Size evaluation of gold nanoparticles by UV-VIS spectroscopy. J. Phys. Chem., 113(11): 4277-4285. https://doi.org/10.1021/jp8082425.
Anthonsen, M. W., Varum, K. M., Hermansson, A. M., Smidsrod, O. and Brant, D. A. 1994. Aggregates in acidic solutions of chitosans detected by static laser light scattering. Carbohydr. Polym., 25(1): 13-23. https://doi. org/10.1016/0144-8617(94)90157-0.

Dutta, A., Paul, A. and Chattopadhyay, A. 2016. The effect of temperature on the aggregation kinetics of partially bare gold nanoparticles. RSC Adv., 6(85): 82138-82149. https:// doi.org/10.1039/C6RA17561A.

Georgiev, P., Bojinova, A., Kostova, B., Momekova, D., Bjornholm, T. and Balashev, K. 2013. Implementing atomic force microscopy (AFM) for studying kinetics of gold nanoparticle's growth. Colloids Surf. A Physicochem. Eng. Asp., 434: 154-163. https://doi.org/10.1016/j.colsurfa. 2013.05.064.

Huang, H. and Yang, X. 2004. Synthesis of chitosanstabilised gold nanoparticles in the absence/presence of tripolyphosphate. Biomacromolecules, 5(6): 2340-2346. https://doi.org/10.1021/bm0497116.

Lakshmi Narayanan, R. and Sivakumar, M. 2014. Preparation and characterization of gold nanoparticles in chitosan suspension by one-pot chemical reduction method. Nano Hybrids, 6: 47-57. https://doi.org/10.4028/www.scientific. net/NH.6.47.

Mazzola, L. 2003. Commercializing nanotechnology. Nat. Biotechnol., 21(10): 1137-1143. https://doi.org/10.1038/ nbt1003-1137.

Mohan, C. O., Gunasekaran, S. and Ravishankar, C. N. 2019. Chitosan-capped gold nanoparticles for indicating temperature abuse in frozen stored products. NPJ Sci. Food., 3(1): 1-6. https://doi.org/10.1038/s41538-019-0034-z. 
Mulvaney, P. 1996. Surface plasmon spectroscopy of nanosised metal particles. Langmuir, 12(3): 788-800. https://doi. org/10.1021/la9502711.

Osman, Z. and Arof, A. K. 2003. FTIR studies of chitosan acetate based polymer electrolytes. Electrochim. Acta, 48(8): 993-999. https://doi.org/10.1016/S0013-4686 (02)00812-5.

Patil, M. P. and Kim, G. D. 2018. Marine microorganisms for synthesis of metallic nanoparticles and their biomedical applications. Colloids Surf. B., 172: 487-495. https://doi. org/10.1016/j.colsurfb.2018.09.007.

Rinaudo, M. 2006. Chitin and chitosan: Properties and applications. Prog. Polym. Sci., 31(7): 603-632. https://doi. org/10.1016/j.progpolymsci.2006.06.001.

Simeonova, S., Georgiev, P., Exner, K. S., Mihaylov, L., Nihtianova, D., Koynov, K. and Balashev, K. 2018. Kinetic study of gold nanoparticles synthesized in the presence of chitosan and citric acid. Colloids Surf. A Physicochem. Eng. Asp., 557: 106-115. https://doi.org/10.1016/j.colsurfa. 2018.02.045.

Sun, L., Li, J., Cai, J., Zhong, L., Ren, G. and Ma, Q. 2017. One pot synthesis of gold nanoparticles using chitosan with varying degree of deacetylation and molecular weight. Carbohydr. Polym., 178: 105-114. https://doi. org/10.1016/j.carbpol.2017.09.032.

Watzky, M. A. and Finke, R. G. 1997. Transition metal nanocluster formation kinetic and mechanistic studies. A new mechanism when hydrogen is the reductant: slow, continuous nucleation and fast autocatalytic surface growth. J. Am. Chem. Soc., 119(43): 10382-10400. https:// doi.org/10.1021/ja9705102.

Date of Receipt $\quad: 18.02 .2021$

Date of Acceptance : 15.06.2021 